\title{
Expression, purification, refolding, and characterization of octreotide-interleukin-2: A chimeric tumor-targeting protein
}

\author{
JING JIANG ${ }^{1}$, LEIXIU DENG ${ }^{2}$, LICHUN HE ${ }^{2}$, HAITING LIU ${ }^{2}$ and CHANGHAI WANG ${ }^{1}$ \\ ${ }^{1}$ School of Life Science and Biotechnology, Dalian University of Technology, Dalian 116024; \\ ${ }^{2}$ Shandong Simcere-Medgenn Biopharmaceutical Co., Ltd., P.R. China
}

Received April 15, 2011; Accepted May 18, 2011

DOI: 10.3892/ijmm.2011.723

\begin{abstract}
The targeting of tumor cells by peptides for drug delivery is a promising strategy for cancer therapy. Interleukin-2 (IL-2), which mediates anti-tumor cellular immune responses, has been approved as a therapy for cancer. However, the serious side effects and short half-life of recombinant IL-2 (rIL-2) has limited its use clinically. Somatostatin receptors (SSTRs), which are expressed in a large number of human tumors, are the targets for in vivo tumor targeting. In this study, we have constructed and expressed a novel chimeric recombinant protein containing octreotide analogs and IL-2 in order to target the SSTR binding with tumor cells. The fusion protein somatostatin receptor targeted interleukin-2 (SIL), which was purified from bacterial inclusion bodies and refolded with high purity, was analyzed by sodium dodecyl sulfate-polyacrylamide gel electrophoresis and reverse-phase high-performance liquid chromatography. Tryptophan emission fluorescence was used to measure the structural changes in SIL after renaturation. Cell proliferation experiments showed that this chimeric protein retained the biological activities of hIL-2. Furthermore, the tumor binding capacity of SIL acting through SSTRs was shown through co-immunoprecipitation. Competition binding with octreotide of tumor cells also confirmed that SIL binds to tumor cells through the target peptide octreotide. Moreover, the performance of SIL in stimulating the proliferation of lymphocyte cell lines after binding to tumor cells showed that the immunocytokine, SIL, retained its bioactivity at the tumor site. These results suggest that SIL is a recombinant fusion protein that may be used for tumor-targeted drug delivery.
\end{abstract}

\section{Introduction}

Targeted cancer therapies, which are designed to sacrifice cancer cells with little damage to normal healthy cells, are a huge part of current cancer research. These therapies result

Correspondence to: Dr Jing Jiang, School of Life Science and Biotechnology, Dalian University of Technology, Dalian 116024, P.R. China

E-mail: jing_jiang1974@sina.com

Key words: interleukin-2, octreotide, somatostatin receptor, expression, purification, refolding, tumor target, bioactivity in less side effects and a better quality of life for the patients (1). The selective delivery of bioactive agents (e.g., cytotoxic drugs, radionuclides, or immunostimulatory cytokines) to the tumor site represents one of the most promising strategies, which has unprecedented efficacy and tolerability, in the development of diagnostic and anticancer therapies (2-4). Using the strong antineoplastic activities of peptide-guided tumor-targeting cytokines and tumor-targeting immunocytokines in preclinical models of solid cancers as an example (5-9), we constructed a peptide-guided interleukin-2 (IL-2) chimeric protein that could particularly enrich tumor sites with immunocytokines.

IL-2, which was the first interleukin molecule discovered, can stimulate the proliferation of T cells, B cells, and primary human natural killer (NK) cells when it is bound to its receptor. IL-2 is thought to mediate antitumor cellular immune responses by enhancing the cytotoxicity of $\mathrm{CD}^{+}$ $\mathrm{T}$ cells and NK cells, and these effects have resulted in the approval of IL-2 as a therapy for cancer (10). Although the systemic administration of IL-2 has been shown to stimulate antitumor responses in vivo, its efficacy in the clinic has been limited by the development of serious side effects and its short half-life $(11,12)$. These deficiencies of IL-2 have resulted in an urgent need for the development of an improved recombinant human IL-2 (rhIL-2) for wide clinical use. The conjugation of polyethylene glycol to rhIL-2 resulted in a polyethylene glycol-modified IL-2 (PEG-IL-2), which exhibited a markedly prolonged circulating half-life (13). The construction of monoclonal antibody/IL-2 fusion proteins, which can directly target this potent cytokine to tumors, is a promising strategy for the immunotherapy of both solid and lymphoid malignancies (14).

Somatostatin (SST), which was first discovered in hypothalamic extracts, was identified as a hormone that inhibited the secretion of growth hormone. SST is secreted from a broad range of tissues, including pancreas, the intestinal tract, and regions of the central nervous system outside of the hypothalamus (15). SST acts by binding to a family of five SST receptors (SSTR 1-5), all of which are G protein-coupled receptors (16). Subsequently, SSTRs were found to secrete from a wide range of tissues, including lymphoid cells, diabetic islets, and the nervous and gastro-entero-pancreatic systems (17-19). In particular, different SSTR 1-5 subtypes are expressed in tumor cells depending on the type of tumor (20-24). Several studies have shown that SST has potential antitumor activities because 
it can bind to SSTRs in several human cancers in vitro and in vivo $(25,26)$. Consequently, SSTRs may be a potential target for the delivery of tumor-specific drugs. Some SST analogs, including octreotide, lanreotide, and BIM23014, possess the same pharmacological properties as SST, except that they are cleared from the circulation at a much slower rate (27). These analogs are most frequently used as radiopharmaceuticals for therapeutic and imaging purposes in cancer. However, there have been no studies on the peptide-guiding efficacy of SST analogs that are fused with protein cytokines. The construction of an SST analog to guide the delivery of IL-2 may provide a potent cancer therapy option.

Somatostatin receptor targeted interleukin-2 (SIL), which is a recombinant chimeric protein made up of octreotide and rhIL-2, is a biofunctional protein with dual receptor binding specificities. This chimeric protein can offer tissue targeting in clinical therapy. Biofunctional SIL can be targeted to bind to SSTR in tumor cells and activate T cells with specific binding with IL-2. Bridging T cells to neoplastic cells with a biofunctional fusion protein could facilitate interactions between tumors and $\mathrm{T}$ cells. This strategy can provide a new way to treat cancer. In addition, the fusion protein may increase both of the half-lives of IL-2 and SST, resulting in improved therapeutic potentials. In order to study this new biofunctional fusion protein and the dual effects of bridging tumor cells and increasing $\mathrm{T}$ cell proliferation, we constructed, expressed, and purified a chimeric fusion protein using an Escherichia coli expression system and characterized the in vitro biophysical and biofunctional properties of the protein.

\section{Materials and methods}

Plasmid construction of the SIL chimeric protein. The nucleotide sequence corresponding to hIL-2 was previously subcloned into a pET-25b plasmid (Novagen, Madison, WI, USA) between an engineered NdeI site and a HindIII site. This hIL-2 sequence was previously engineered in order to mutate Cys125 to a serine in order to aid in the folding of this cysteine, so that it could participate in native disulfide pairing. In order to construct the chimeric protein, the SIL sequence was amplified using a pair of forward and reverse primers. The sequence of the synthetic forward primer was 5'-CATATGTTCTGTTTCTG GAAGACGTGCACCGGCGGTAGCGCACCTAC-3', which contained an $\mathrm{NdeI}$ site, an octreotide coding sequence, a linker sequence, and a $3^{\prime}$ overhang that overlapped with the $5^{\prime}$ IL-2 sequence. The reverse primer was 5'-CAACACTGACTCA CCATCACCATCACCATTGAAGCTT-3', which contained an appended his6-tag coding sequence for easy purification of the recombinant protein and a $3^{\prime}$ HindIII site that was preceded by a stop codon. The SIL sequence was cloned into the pET-25b vector between the NdeI and HindIII sites. The plasmid was screened in DH5a and then expressed in BL21 (DE3) (Novagen). The sequence of the resulting plasmid pET25b-octreotide-hIL-2 was confirmed by DNA sequencing (Invitrogen, Shanghai).

Cell culture and fermentation. The recombinant protein that was expressed by the transformed bacteria was cultured in LB broth supplemented with $100 \mu \mathrm{g} / \mathrm{ml}$ of ampicillin at $37^{\circ} \mathrm{C}$ in an incubator shaker. The E. coli BL21 (DE3) harboring pET25b-
octreotide-IL-2 was then grown in a 5-L bioreactor (Biostat B5 B. Braun), fed by batch fermentation in synthetic medium at $37^{\circ} \mathrm{C}$, and induced at an OD600 of about 25 at $37^{\circ} \mathrm{C}$ with $1 \mathrm{mM}$ isopropyl $\beta$-D-1-thiogalactopyranoside (IPTG). When the cultures achieved an OD600 of about 60 , the cells were harvested with centrifugation at $10,000 \mathrm{x} \mathrm{g}$ for $10 \mathrm{~min}$. The expression yield was analyzed by sodium dodecyl sulfatepolyacrylamide gel electrophoresis (SDS-PAGE), which was followed by Coomassie brilliant blue staining.

Inclusion body washing and protein purification. The harvested cell paste was resuspended in buffer containing $20 \mathrm{mM}$ Tris-HCl, $5 \mathrm{mM}$ ethylenediaminetetraacetic acid (EDTA), $\mathrm{pH} 8.0$, and the SIL inclusion bodies were extracted with a high-pressure homogenizer under a pressure of 60-80 MPa. The whole process was repeated three times. The pellet for the inclusion bodies was collected by centrifugation (20,000 x g; $20 \mathrm{~min}$ ) and then resuspended and washed in $2 \mathrm{M}$ urea, containing $20 \mathrm{mM}$ Tris- $\mathrm{HCl}, 50 \mathrm{mM} \mathrm{NaCl}, 2 \%$ Triton $\mathrm{X}-100$, and $0.5 \mathrm{mM}$ 1,4-dithiothreitol (DTT), pH 8.0. Finally, the pellet was washed with $20 \mathrm{mM}$ Tris- $\mathrm{HCl}, \mathrm{pH}$ 8.0, which was followed by centrifugation. The inclusion bodies were solubilized in a buffer containing $8 \mathrm{M}$ urea, $50 \mathrm{mM}$ Tris, pH 8.0, $300 \mathrm{mM}$ $\mathrm{NaCl}, 10 \mathrm{mM}$ imidazole and $10 \mathrm{mM}$ 2-mercaptoethanol.

Isolation of the target protein was achieved by immobilized metal affinity chromatography (IMAC) with an AKTA Explore 100 system. Urea buffer (8 M) containing $<80 \mathrm{mM}$ imidazole was used to wash the non-specifically bound protein off of the column packed with chelating sepharose resin charged with nickel ions. The recombinant SIL was eluted with buffer containing $200 \mathrm{mM}$ imidazole.

Refolding and production of SIL. DTT was added to the IMAC-purified chimeric protein in order to produce a final concentration of $30 \mathrm{mM}$ to dissociate mismatched disulfide bonds. The refolding buffer containing $20 \mathrm{mM}$ Tris, $100 \mathrm{mM}$ arginine, $0.4 \%$ Tween- $80(\mathrm{v} / \mathrm{v}), 2 \mathrm{mM}$ EDTA and $10 \mathrm{mM}$ L-Cysteine at $\mathrm{pH} 8.0$ was then added drop-wise to the reduced chimeric protein. Upon completion of the 4-fold dilution, the mixture was adjusted to a $\mathrm{pH}$ of 8.6 , the concentration of the chimeric protein was adjusted to $0.1-0.3 \mathrm{mg} / \mathrm{ml}$, and the mixture was then allowed to stir slowly overnight. After $\sim 18 \mathrm{~h}, 5 \mu \mathrm{M}$ $\mathrm{CuCl}_{2}$ was added and stirred for $2 \mathrm{~h}$ in order to catalyze the oxidation of free cysteines. The mixture was then concentreated using an Amicon Stirred Cell 8200 (Millipore, Billerica, MA, USA) fitted with a Millipore Ultrafiltration Membrane YM filter (NMWL 3000). The concentrated chimeric protein was dialyzed against $20 \mathrm{mM}$ sodium phosphate buffer ( $\mathrm{PB}$; $\mathrm{pH} 8.0)$ and then centrifuged at $10,000 \mathrm{x} \mathrm{g}$ for $10 \mathrm{~min}$ in order to remove the precipitation that formed from the misfolded protein. Refolded SIL was purified using a Ni-NTA Superflow (Qiagen $\mathrm{GmbH}$, Hilden, Germany) column, which was pre-equilibrated in a PB-balanced buffer containing $150 \mathrm{mM} \mathrm{NaCl}$ and $10 \mathrm{mM}$ imidazole, $\mathrm{pH}$ 8.0. The column was then eluted with 50, 100 and $150 \mathrm{mM}$ imidazole in balanced buffer. After identification by non-reducing SDS-PAGE, the high-purity fraction of the target SIL monomer was then refined by High Q Sepharose in order to remove the endotoxin. The protein concentration was determined by absorbance at $280 \mathrm{~nm}$, and this material was used for the experiments described in this study. 
SDS-PAGE and Western blot analysis. Samples for SDS-PAGE were resuspended in SDS sample buffer that was reduced (containing 2-mercaptoethanol) or non-reduced (without 2-mercaptoethanol), boiled for $3 \mathrm{~min}$, and then loaded onto gels. The proteins were detected by Coomassie Brilliant staining or silver staining and then transferred to a nitrocellulose membrane under constant voltage for the Western blot analysis, which was performed according to a previously published protocol (28). Primary antibodies, including IL-2 and SSTR2 antibodies, and secondary antibodies (Santa Cruz Biotechnology, Inc., Santa Cruz, CA, USA) were diluted in PBST (phosphate-buffered saline containing $0.02 \%$ Tween-20) and applied during a washing step. Proteins were detected using the Amersham ECL Western blotting detection kit (GE Healthcare, Piscataway, NJ, USA) and exposed on photographic film. All antibodies were from Santa Cruz Biotechnology, Inc.

Reverse phase high-performance liquid chromatography analysis. For the high-performance liquid chromatography (HPLC) analysis, an Agilent 1200 was used. The analytical reverse-phase C18 column was from Vydac Co., Ltd. Trifluoroacetic acid and acetonitrile, which were of chromatographic grade, were from Amresco. Reduced and non-reduced SIL proteins were analyzed using the following gradients and buffers: from $64 \% \mathrm{~A}$ (water, $0.05 \%$ trifluoroacetic acid) to $64 \%$ $\mathrm{B}$ (acetonitrile, $0.05 \%$ trifluoroacetic acid) in $28 \mathrm{~min}$.

Mass spectrometry. Mass spectrometric analyses were carried out on an electrospray ionization/time-of-flight (ESI/TOF) mass spectrometer (MS) from Bruker Daltonics, which was equipped with an electrospray ionization system. The instrument was operated in a positive ion mode, and mass spectra were recorded with an $\mathrm{m} / \mathrm{z}$ range of 20-4000. All mass spectra were recorded as centroid in real time. The capillary voltage was maintained at $-4500 \mathrm{~V}$ with the end plate offset at $-500 \mathrm{~V}$. The gas flow to the nebulizer was set at a pressure of 0.4 bars, while the drying temperature and the drying gas flow rate were $200^{\circ} \mathrm{C}$ and $5.0 \mathrm{l} / \mathrm{min}$, respectively. Both denatured and refolded SIL dissolved in buffer were injected directly into the ESI-TOF MS for analysis, and the injection volume was less than $5 \mu$. Calibration was performed using sodium trifluoroacetate. The mass spectra were analyzed using the MicrOTOF control software supplied with the MS. Each analyte ion was measured by summing the entire peak in the total ion chromatogram, and the sample mass was finally obtained by deconvolution in the data analysis from the MicrOTOF control software.

Measurements of tryptophan emission fluorescence. The tryptophan (Trp) emission fluorescence of SIL was measured with a Hitachi F-4500 spectrophotometer using cuvettes with an optical path length of $1 \mathrm{~cm}$. The temperature was maintained at a constant $20^{\circ} \mathrm{C}$ using an external bath circulator. Trp emission fluorescence was measured at an excitation wavelength of $270 \mathrm{~nm}$. The emission spectra were measured from 300 to $400 \mathrm{~nm}$ (excitation and emission slit width, $5 \mathrm{~nm}$ ). The scan speed was $240 \mathrm{~nm} / \mathrm{min}$. The concentration of protein was $0.9 \mu \mathrm{M}$ in $10 \mathrm{mM}$ Tris-HCl buffer (pH 7.4).

Cytotoxic T lymphocyte cell line proliferation assays. The specific activities of SIL and IL-2 were assayed by measuring the proliferation of IL-2-dependent murine cytotoxic T lymphocyte lines (CTLL-2; ATCC, Manassas, VA, USA) according to the methods described by Weston (29). Seven serial two-fold dilutions of rhIL-2 standard (Sigma-Aldrich, Co., St. Louis, MO, USA) were made with three replicates in each dilution. The resulting concentration of rhIL-2 ranged from 200 to $6.25 \mathrm{IU} / \mathrm{ml}$ in each well, and the SIL was diluted to the same concentration as rhIL-2 based on the molar concentration. The cells were incubated for $24 \mathrm{~h}$, and 3-(4,5-dimethylthiazol2-yl)-2,5-diphenyltetrazolium bromide (MTT) was added and then solubilized in $10 \% \mathrm{SDS}$ overnight at $37^{\circ} \mathrm{C}$. The next day, the optical density of the culture medium was quantified by reading the absorbance at $630 \mathrm{~nm}$. The relative growth rate (RGR) of the cell proliferation was calculated according to the following formula, and the results were expressed as an average of three samples \pm the standard error: RGR (\%) $=[($ Absorbance of sample - Absorbance of negative control $) /$ Absorbance of negative control] x 100.

Tumor target receptor investigation. SSTR2 expression in different cell lines was investigated by Western blotting. The cell lines chosen for SSTR2 detection included normal liver cell line L-02, mouse hepatoma cell line $\mathrm{H} 22$, and several kinds of human tumor cell lines, including the human breast cancer cell line MDA-MB-231, the human pancreatic carcinoma cell line PANC-1, the human hepatocellular carcinoma cell line SMMC7721, the human gastric adenocarcinoma epithelial cell line AGS and the gastric cancer cell line SGC7901. All cell lines used in the experiments were from ATCC or CCTCC (China Center for Type Culture Collection). The cells were cultured, suspended, and adjusted to the same concentration of cell number, and then lysed, mixed with SDS-PAGE loading buffer. SDS-PAGE and Western blotting methods were carried out as described above. Primary and secondary antibodies for SSTR2 were from Santa Cruz Biotechnology, Inc.

Co-immunoprecipitation (Co-IP) evaluation of the binding capacity of SIL to tumor cells. The tumor cells that expressed SSTR were grown in RPMI-1640 supplemented with $10 \%$ fetal bovine serum and then divided into three groups, each containing $1 \times 10^{7}$ cells. PBS and equal doses of rhIL-2 and SIL were added to each group. After being incubated for $4 \mathrm{~h}$, all of the groups of cells were washed with PBS three times and then harvested and lysed in buffer $(150 \mathrm{mM} \mathrm{NaCl}, 1 \%$ IGEPAL CA-630 (Sigma), 0.5\% sodium deoxycholate, $0.1 \%$ SDS, $50 \mathrm{mM}$ Tris- $\mathrm{HCl}, \mathrm{pH} 8.0$, and $1 \mathrm{mM}$ phenylmethylsulfonyl fluoride) based on the reference reported by Yaciuk (30).

The SSTR antibody was added to the cell lysates and then incubated at $4^{\circ} \mathrm{C}$ for $4 \mathrm{~h}$. Protein A Sepharose beads (GE Healthcare) were then added to the mixture and incubated for $1 \mathrm{~h}$ at $4^{\circ} \mathrm{C}$ in order to pull down antibody-specific complexes, and the precipitate was washed five times with $1 \mathrm{ml}$ of RIPA buffer (20 mM Tris-HCl, pH 7.5, $150 \mathrm{mM} \mathrm{NaCl}, 1 \mathrm{mM} \mathrm{Na}_{2}$ EDTA, 1 mM EGTA, 1\% NP-40, 1\% sodium deoxycholate, $2.5 \mathrm{mM}$ sodium pyrophosphate, $1 \mathrm{mM}$ b-glycerophosphate, $1 \mathrm{mM} \mathrm{Na}_{3} \mathrm{VO}_{4}, 1 \mu \mathrm{g} / \mathrm{ml}$ leupeptin). The final precipitate was boiled in protein loading buffer for $3 \mathrm{~min}$ and then run on 15\% SDS-PAGE for Western blot analysis. The IL-2 antibody and the horseradish peroxidase (HRP)-coupled secondary anti- 
body used in the Western blotting were purchased from Santa Cruz Biotechnology, Inc.

SIL bioactivity evaluation of competitive binding with octreotide to tumor cells. The tumor cells used in this experiment were SGC7901 cells. After routine passage cultures of SGC7901 in vitro, the cells were seeded to 24 -well plates. When the plate was covered by the cells, a concentration gradient of rhIL-2 and SIL, with final concentrations of 2,000, 1,000 and $500 \mathrm{IU} / \mathrm{ml}$ was added to the plate, respectively. Different doses of octreotide that was competitively bound to SGC7901 tumor cells with SIL $(2,000 \mathrm{IU}$ SIL $+100 \mu \mathrm{g} / \mathrm{ml}$ OCT, $2,000 \mathrm{IU} \mathrm{SIL}+50 \mu \mathrm{g} / \mathrm{ml}$ OCT, 2,000 IU SIL $+10 \mu \mathrm{g} / \mathrm{ml} \mathrm{OCT,} \mathrm{2,000} \mathrm{IU} \mathrm{SIL}+1 \mu \mathrm{g} / \mathrm{ml}$ OCT) were carried out in another plates. A negative control group of PBS was set. Each group had three parallels.

The supernatant, which was discarded $4 \mathrm{~h}$ after the drugs were added to the tumor cells, was washed three times with PBS. At this stage, PBS had to be discarded completely. Subsequently, a wash step with RPMI-1640 medium was performed, and CTLL-2 cells (without IL-2) were then added to the plate. The plate was incubated overnight, observed, and photographed on the second day. The supernatant was transferred to another plate, and $100 \mu \mathrm{l}$ of MTT was added. SDS-HCl was added after a $4 \mathrm{~h}$ incubation period. The supernatant was then incubated overnight again. Finally, an absorbance value at $570 \mathrm{~nm}$ was used to determine the rate of CTLL-2.

\section{Results}

Plasmid construction and expression of SIL. The results of the DNA sequencing analysis demonstrated that the cDNA of octreotide/hIL-2 was inserted into the $E$. coli expression vector pET25b. Additionally, a His6-tag was added to the C-terminus in order to simplify the purification of the target protein. Feed batch fermentation of the positive clone was conducted on a 5-L bioreactor at $37^{\circ} \mathrm{C}$ and induced at an OD600 of 25.3 at $37^{\circ} \mathrm{C}$ with $1 \mathrm{mM}$ IPTG. The culture cells were sampled every hour after induction and tested on 15\% SDS-PAGE at the end of fermentation (Fig. 1A). The cultures achieved an OD600 of 76 after a nearly 4-h induction. The wet weight of the bacteria per liter of culture was $105 \mathrm{~g}$, and they were then harvested with centrifugation at $10,000 \mathrm{x}$ g for $10 \mathrm{~min}$. The expression levels of SIL protein, which were determined by gel densitometry, were $28.3 \%$ of the total cellular protein (data not shown).

Purification of SIL from inclusion bodies by IMAC under denaturing and reducing conditions. Inclusion bodies containing SIL were washed several times with Tris- $\mathrm{HCl}$ buffer containing $2 \mathrm{M}$ urea and 2\% Triton X-100 in order to remove soluble background proteins. During the washing process, the samples at each step were visualized by reduced SDS-PAGE and Coomassie Blue staining (Fig. 1B). Slightly more protein could be dissolved in the wash buffer, which contained urea and Triton X-100, according to the SDS-PAGE test. Finally, more than $1 \mathrm{~g}$ of the inclusion bodies could be separated from $10 \mathrm{~g}$ of bacteria with a purity of nearly $50 \%$. The inclusion bodies were then solubilized in a urea-containing buffer, and denatured SIL was effectively separated from the bulk of the background proteins by Ni-NTA (Qiagen $\mathrm{GmbH}$ ) with a yield of $30 \mathrm{mg} / \mathrm{g}$ of bacteria. The purity was nearly $90 \%$ when analyzed by SDS-PAGE.
A

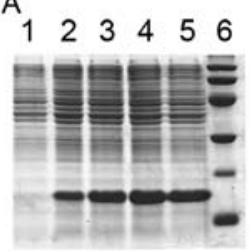

C
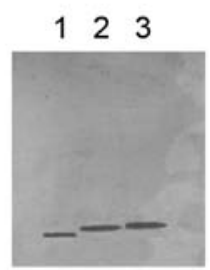

B $\begin{array}{llllllll}1 & 2 & 3 & 4 & 5 & 6 & 7 & 8\end{array}$
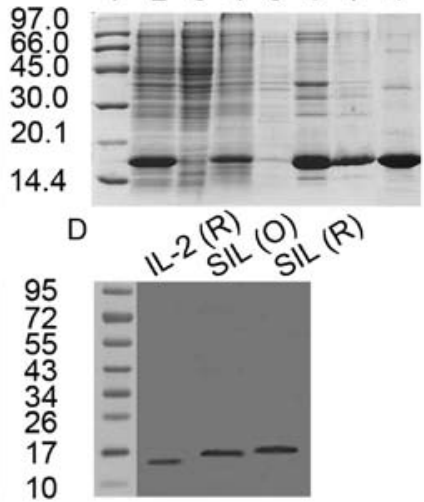

Figure 1. Results of sodium dodecyl sulfate-polyacrylamide gel electrophoresis (SDS-PAGE) illustrating the fermentation on a bioreactor and the purification of SIL. Coomassie Blue staining or silver staining of the gels was performed following resolution of the samples by electrophoresis. (A) Lane 1, non-induced whole cell lysate; lanes 2-5, whole cell lysate induced with $1 \mathrm{mM} \mathrm{IPTG}$ at $37^{\circ} \mathrm{C}$ for 1, 2, 3, $4 \mathrm{~h}$, respectively; lane 6, low molecular weight (MW) markers. (B) Lane 1, MW markers; lane 2, whole cell lysate of fermentation after being induced for $4 \mathrm{~h}$; lane 3, supernatant of homogenesis buffer after centrifuge; lane 4, SIL inclusion bodies (IB) washing supernatant of $2 \mathrm{M}$ urea and $2 \%$ Triton X-100 containing $50 \mathrm{mM} \mathrm{NaCl}$ and $0.5 \mathrm{mM}$ of 1,4-dithiothreitol (DTT), pH 8.0; lane 5, SIL IB washing supernatant of $20 \mathrm{mM}$ Tris- $\mathrm{HCl}, \mathrm{pH} 8.0$; lane 6, precipitate of final wash of SIL inclusion bodies; lane 7, SIL IB dissolved in $8 \mathrm{M}$ urea containing 2 mM DTT, pH 8.0; lane 8, purified recombinant SIL protein under reduced conditions. (C) Lane 1, recombinant human IL-2 standard (Sigma-Aldrich Co.); lane 2, purified and refolded recombinant SIL protein under non-reducing conditions; lane 3 , same sample as lane 2 under reducing conditions. (D) Western blot analysis of refolded SIL compared with recombinant hIL-2.

Preparation of active SIL by refolding using air oxidation. After concentration, dialysis, and filtration, the final yield was nearly $6 \mathrm{mg} / \mathrm{g}$ of bacteria. This represented a loss of $80 \%$ of the protein, which was mostly due to precipitation. The relative purity of the refolded SIL was visualized by both SDS-PAGE and Western blotting (Fig. 1C and D). The molecular weight (MW) of SIL was nearly $17 \mathrm{kDa}$, the mobility of SIL on SDS-PAGE was slower than that of IL-2 for the additional targeting peptide, and the mobility of refolded SIL in non-reduced SDS-PAGE was faster than that in the reduced SDS-PAGE. The purity of the target chimeric protein was beyond $95 \%$, as determined by reverse phase-HPLC (RP-HPLC). The reaction time $\left(t_{R}\right)$ of the refolded SIL on a C18 analysis column was $2.8 \mathrm{~min}$ ahead of that of the denatured one under the same chromatographic conditions. The MS analysis of the denatured SIL protein resulted in a MW of 17,574 Da, while the refolded SIL protein was $17,570 \mathrm{Da}$, which was $4 \mathrm{Da}$ less than that of the denatured protein. Because there are 4 cysteines in the SIL protein, 4 hydrogen atoms were removed from the molecular protein after disulfide bond formation. The Trp emission fluorescence spectrum of the refolded SIL showed that the maximum emission wavelength was at $316 \mathrm{~nm}$ when excited at $270 \mathrm{~nm}$, while the maximum emission wavelength of denatured SIL was at $340 \mathrm{~nm}$, which demonstrated a very compact tertiary structure. Furthermore, the refolded SIL had the same stimulation potency as CTLL-2 cells and rhIL-2. The characterized results of the refolding are shown in Fig. 2.

Investigation of the expression levels of SSTR in order to choose a targeting verification model. Cell lines that were chosen 
A

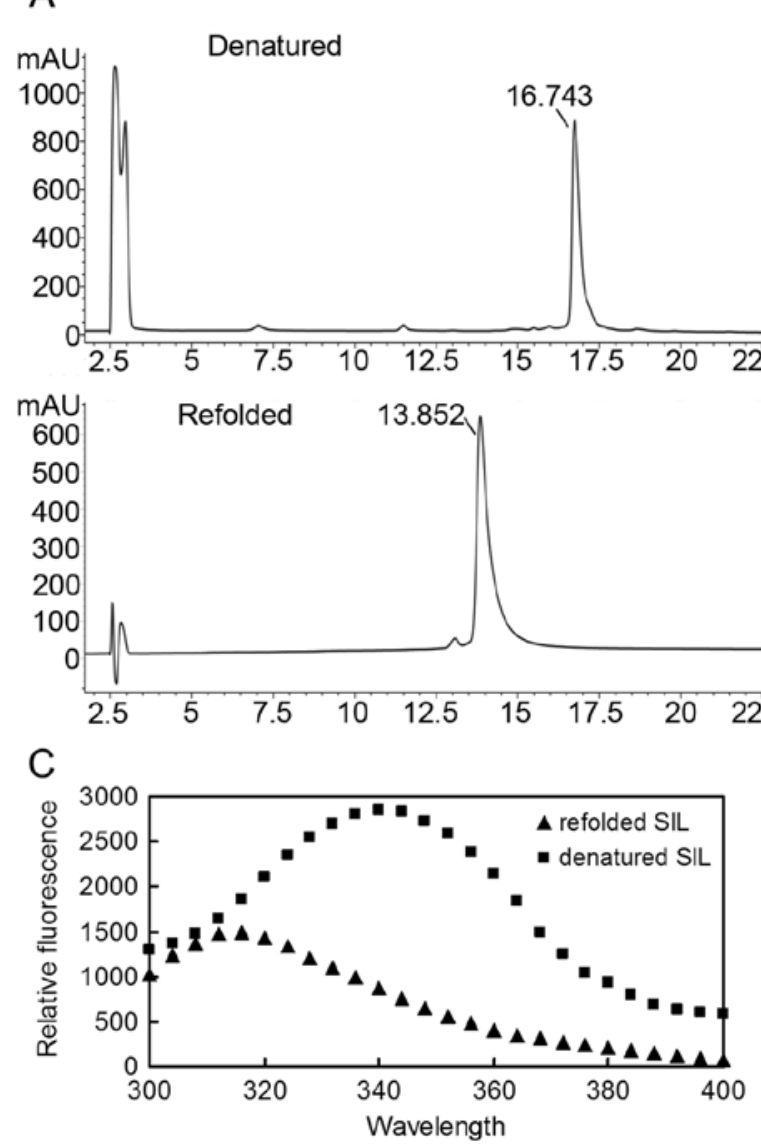

B

Intens

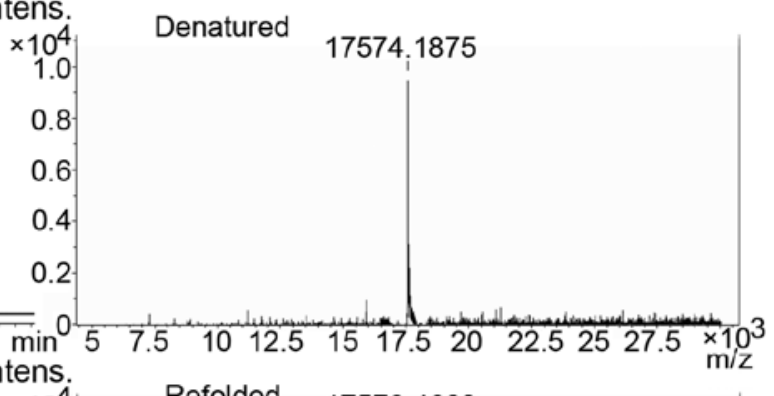

$\times 10^{4} \quad$ Refolded $\quad 17570.4688$

2.0

1.0 0.5
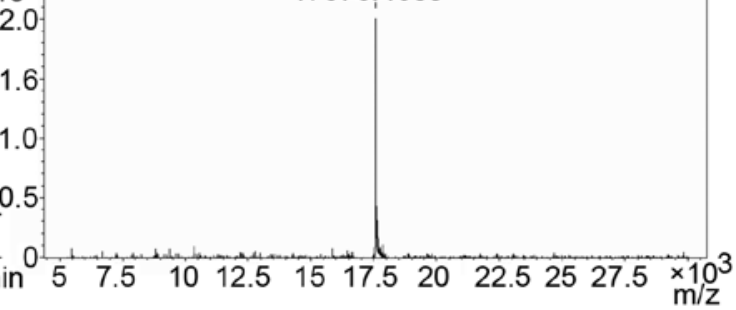

D

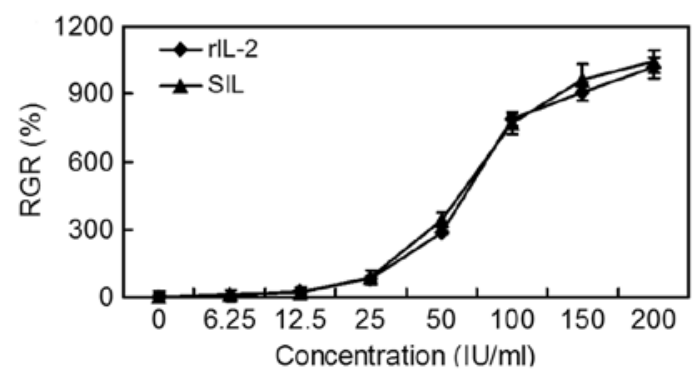

Figure 2. Characterization to evaluate the correct refolding of the recombinant SIL protein. (A) HPLC profile of the denatured and refolded SIL by an analytical RP-C18 column using the following gradient and buffers: from $64 \%$ A (water, $0.05 \%$ trifluoroacetic acid) to $64 \%$ B (acetonitrile, $0.05 \%$ trifluoroacetic acid) in 28 min. (B) Deconvoluted mass spectra of the SIL denatured protein and refolded protein showed that the accurate MWs were 17,574.1875 and 17,570.4688 Da, respectively. (C) Tryptophan emission fluorescence spectrum of refolded and denatured SIL (excitation wavelength at $270 \mathrm{~nm}$ ). (D) CTLL-2 cell proliferation assay of SIL compared with rhIL-2.

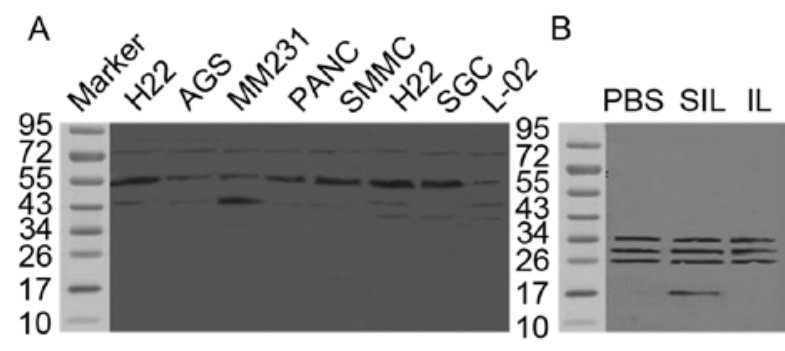

Figure 3. Immunoblotting investigation of the levels of expression of the somatostatin receptor, subtype 2 (SSTR) with the SSTR2 antibody in the test cell lines, including the mouse hepatoma cell lines H22 and the human tumor cell lines, human gastric adenocarcinoma epithelial cell lines AGS, human breast cancer cell lines MDA-MB-231 (MM231), human pancreatic carcinoma cell lines PANC-1 (PANC), human hepatocellular carcinoma cell lines SMMC7721 (SMMC), the gastric cancer cell SGC 7901 (SGC), and the human normal liver cells L-02. The SIL target binding to tumor cells through SSTR is illustrated. (A) SSTR2 content was evaluated using Western blotting. All loaded samples on the gels were from the same number of cells before being transferred to the nitrocellulose membrane. (B) Co-immunoprecipitation results of the evaluation of the binding capacity of SIL to SSTR2 expressing tumor cells of SMMC7721.

for SSTR detection included normal liver cells and several kinds of tumor cell lines, such as H22, SMMC7721, L-02, SGC7901, the breast cancer cell line MDA-MB-231, AGS, and the human pancreatic carcinoma PANC-1. The antibody chosen for analysis was SSTR2 with octreotide or its analogs that were preferentially bound to SSTR2 (21). All of the cell lines that were used for SSTR2 detection were cultured, and their concentrations were adjusted to $1 \times 10^{7}$ cells. As depicted in Fig. 3, H22, SGC7901 and SMMC7721 expressed more SSTR2 with a MW of nearly $55 \mathrm{kDa}$ and PANC, MM231 and AGS expressed less, but more than the normal cell lines L-02, H22, SGC and SMMC7721, which exhibited a distinct overexpression of SSTR2. The tumor cells, SMMC7721 and SGC, which were confirmed to express more SSTR2, were chosen for the SIL target binding test. The results are shown in Fig. 3A.

Evaluation of the SIL binding capacity to tumor cells by Co-IP. The tumor cell line SMMC7721, which was confirmed to express SSTR, was cultured and divided into three groups. PBS and equal doses of rhIL-2 and SIL were added to each group. After incubation and washing, the cells were harvested and lysed in RIPA buffer. The SSTR antibodies were added to the three groups in order to recognize and bind to the receptors. Protein A Sepharose beads were then added in order to capture the antibody-specific complexes. The final precipitate was then boiled in protein loading buffer and run on $15 \%$ SDS-PAGE for Western blot analysis, and an IL-2 antibody 
A
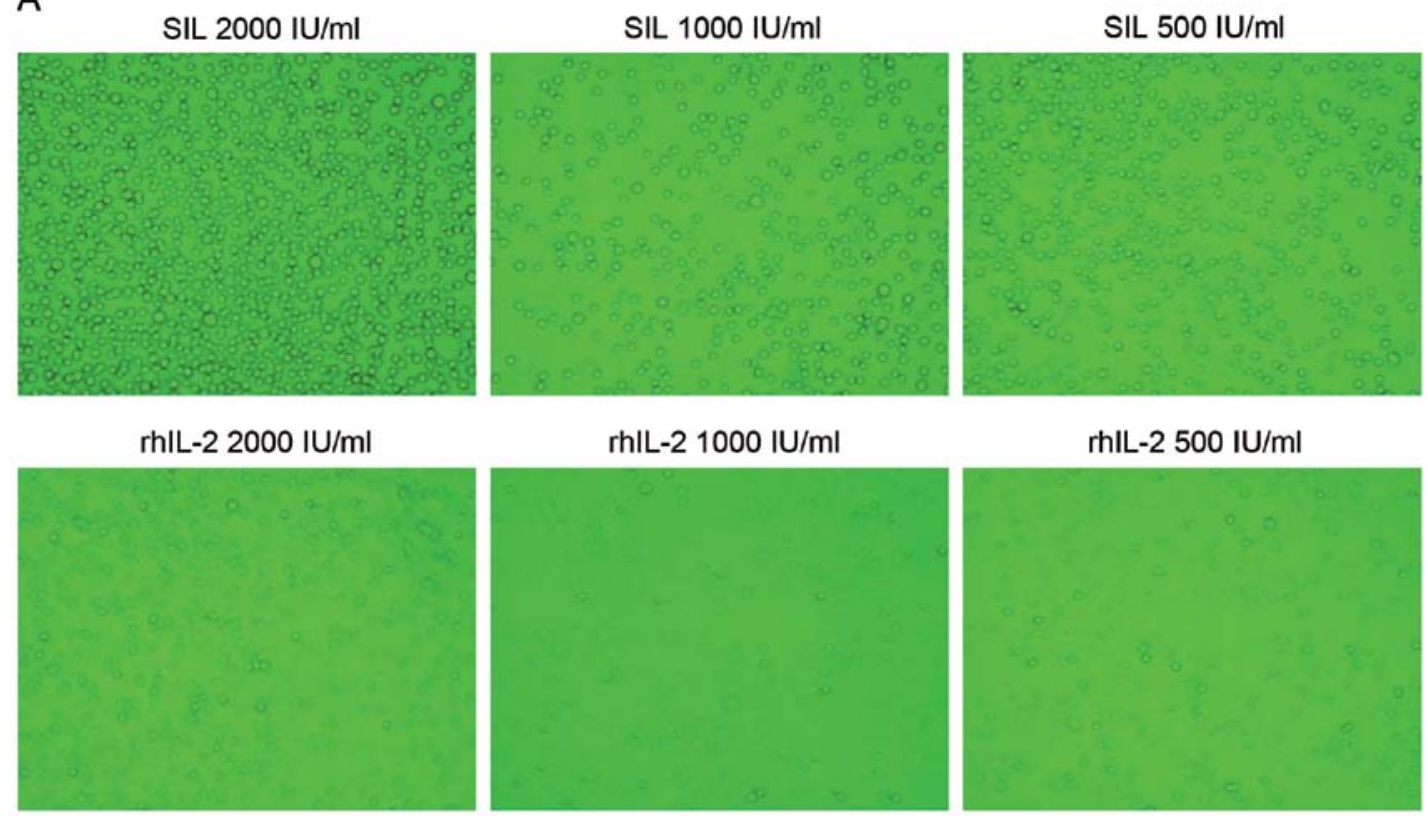

B

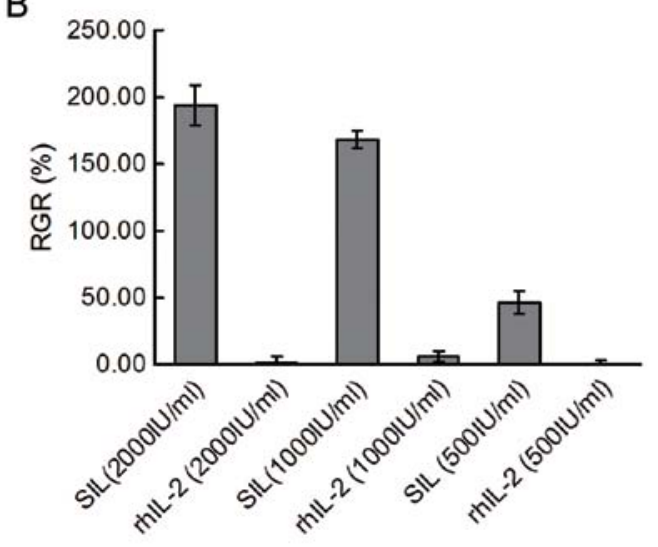

C

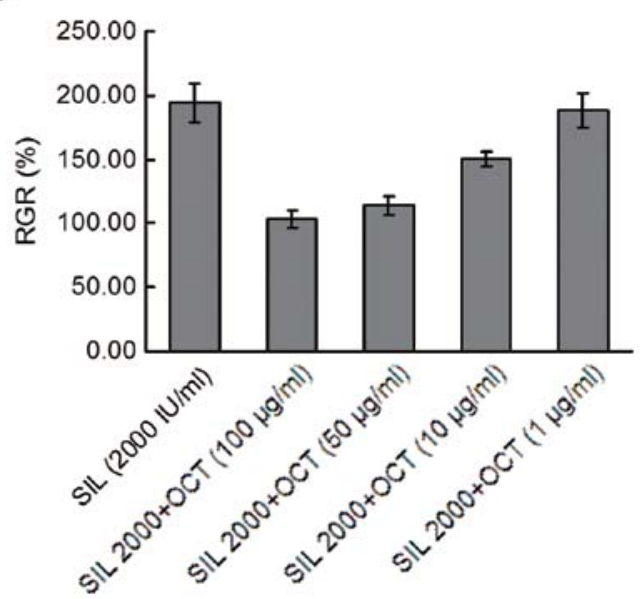

Figure 4. Illustration of the SIL-stimulating bioactivity of CTLL-2 proliferation after binding to SGC7901 cells, and the evaluation of the competitive binding of SIL with octreotide to tumor cells. (A and B) The sequential doses of SIL and IL-2 at concentrations of 2,000, 1,000 and 500 IU/ml were added to the wells with growing SGC7901 cells and incubated in order to bind to each group in two wells. After removing the supernatant, washing, and purging, CTLL-2 cells (without IL-2) were added to each well, incubated overnight, and photographed the second day. The suspended CTLL-2 cells of each well were transferred to another plate for an MTT test, and the absorbance value at $570 \mathrm{~nm}$ was determined in order to calculate the rate of growth of CTLL-2 cells. Data on the relative growth rate of CTLL-2 cells were from two different experiments performed in triplicate. (C) Serial sequential concentrations of the octreotide competitive binding to SGC7901 cells with $2000 \mathrm{IU} / \mathrm{ml}$ SIL were 100, 50, 10 and $1 \mu \mathrm{g} / \mathrm{ml}$, respectively. A negative control group of PBS was set. Data are expressed as mean \pm SE of three independent experiments performed in triplicate ( $\mathrm{P}<0.05$ compared to the control. Each group had three parallels).

and HRP-coupled secondary antibody were used. The results are shown in Fig. 3B. A protein-band ladder and protein bands with MWs that ranged from 26-34 $\mathrm{kDa}$ were detected in all three groups, which was probably due to SSTR antibodies binding to the HRP-coupled secondary antibody. There were also smeared protein bands detected with a MW of $55 \mathrm{kDa}$, which is similar to the MW of SSTR. Only the group of cells to which SIL was added showed a protein band at the MW of nearly $17 \mathrm{kDa}$ with Co-IP. These results illustrate that SIL can bind to tumor cells (SMMC7721) through the SSTR receptor while IL-2 could not bind to the tumor cells.

SIL stimulates the proliferation of CTLL-2 cells after target binding to the tumor cells in vitro and octreotide competitively binds to tumor cells with SIL. The capability of SIL to target bind to the SSTR was confirmed with Co-IP. The bioactivity of the stimulation of the proliferation of CTLL-2 cells should also be examined to assess the chimeric protein binding to the target tumor cells because of the increased probability of three-dimensional structure changes occurring after binding. Therefore, we compared the stimulation capability of CTLL-2 cell proliferation through sequential doses of SIL and rhIL-2 after binding to the tumor cells, SGC7901. In addition, different doses of octreotide were added to another group of SIL-binding tumor cells in order to test the competitive binding of SIL to tumor cells. CTLL-2 cell tests demonstrated that the groups of tumor cells to which SIL was added could still stimulate the CTLL-2 cell proliferation, and this correlated with the dose after washing and changing the culture media, while those groups to which rhIL-2 was added 
did not stimulate the proliferation of CTLL-2 cells (Fig. 4A). The RGR of the SIL group was 20-30 times that of the rhIL-2 group at the same dosage. The competition binding of octreotide to tumor cells was also confirmed with SIL binding to tumor cells with the target peptide octreotide. The results are shown in Fig. 4B and C.

\section{Discussion}

The antibody-based delivery of IL-2 to tumor sites exhibited a promising anti-tumor performance, and this especially enhanced the potency of chemotherapy without apparent additional toxicity (14). The production of such fusion proteins needs a mammalian expression system, which means a high cost of manufacture. The data presented in this study show the rapid production of sufficient quantities of tumor targeting IL-2 by fusion with the SST analog peptide, octreotide, which delivers the immunocytokine to those tumor cells predominantly expressing SSTR2. The purification and refolding steps, which may be further optimized, were shown to be adequate for the production and characterization of this novel protein. Our study demonstrates that this novel chimeric protein can be expressed to a moderate level in a prokaryotic expression system and that active protein can be purified and recovered by dilution into non-denaturing buffer and through air oxidation. Only a single band was seen on both the non-reduced and reduced SDS-PAGE analyses when a purified and correctly refolded SIL sample was tested. The mobility of the SIL protein in the non-reduced SDS-PAGE was faster than that in the reduced SDS-PAGE because of the tight packing of the structure by disulfide bonds, which usually play a very important role in protein stability and bioactivity (31). The refolded SIL with a purity of $95 \%$ was assessed by RP-HPLC with a single peak on the elution graph. In addition, RP-HPLC was used to test the formation of disulfide bonds in the refolded SIL. The retention time of the non-reduced native SIL was faster than that of the reduced denatured SIL because the disulfide bonds resulted in the protein having less exposed hydrophobic patches compared to reduced SIL.

In addition, the MS analysis of SIL resulted in a MW disparity of $4 \mathrm{Da}$ between the denatured protein and the refolded protein, which was due to the disulfide bond formation during the refolding process. Furthermore, the Trp emission fluorescence spectrum of the refolded and denatured SIL demonstrated a very compact tertiary structure of the refolded fusion protein. In addition, satisfactory immunogenicities were shown with Western blotting. The bioactivity test results showed that the recombinant SIL protein that was extracted and purified from inclusion bodies and that was refolded into the native protein possessed the biological activity to stimulate the proliferation of IL-2-dependent CTLL-2 cell lines. Compared with rhIL-2, the biological activity of SIL showed a similar dose-related stimulation of the proliferation of CTLL-2 cells, which indicated that the linkage of octreotide and IL-2 with a glycine-glycine-serine (GGS) amino-acid linker might not disturb the activity of IL-2.

In order to investigate the target binding ability of SIL to tumor cells, SSTR scouting by immunoblotting on several kinds of tumor cells was carried out in order to choose a targeting verification model. SSTRs, which have been reported to be expressed in a large number of human tumors, represent the basis for in vivo tumor targeting $(21,26)$. In this study, H22, SGC7901, and SMMC7721 cell lines were confirmed to express more SSTR2 than other tumor cells and normal liver cells through Western blotting with an SSTR2 antibody. The reasons for choosing an SSTR2 antibody to detect the receptor in immunoblotting were that SSTR2 was reported to be expressed in a majority of tumors, and SST analogs, like octreotide, do not bind with high affinity to all five presently known SSTR subtypes. They have been shown to bind with high affinity to SSTR2 (22).

The data from the Co-IP experiment demonstrate that only the IL-2 with the octreotide tumor targeting peptide could bind to SMMC7721 cells, which have been shown to express SSTR2, while the immunocytokine rhIL-2 alone could not bind to the tumor cells. The results of the Western blotting showed that the lysis of a group of SMMC7721 cells that were incubated with SIL detected a protein at a MW of nearly $17 \mathrm{kDa}$, while the rhIL-2 mixed group and the PBS control groups did not detected the same band, which indicated that only those IL-2 cytokines that were bound to SSTR 2 could be captured from the mixture of the cell lysis buffer system with the SSTR2 antibody. This was presumably due to the SSTR2 antibodies binding to the SSTR2-octreotide-IL2 complex. However, the Western blot analysis result did not exhibit proteins at a weight of $72 \mathrm{kDa}$, which is the MW of the SSTR2octreotide-IL2 complex. A possible explanation for this result is that the complex precipitate included SSTR2-octreotide-IL2 complexes before they were run on SDS-PAGE and boiled in reducing loading buffer that could disassociate the interaction between the SSTR2 and SIL. Most non-covalent-bond binding complexes like antibody-antigen and ligand-receptor have been disaggregated by the SDS detergent. Therefore, the chimeric protein SIL exhibits a target binding potential to tumor cells expressing SSTR2. The bioactivity of SIL after binding to the tumor cells was the key point to be determined since there is an increased probability of three-dimensional structure changing after binding.

Our research has directly shown that the immunocytokine IL-2 possessed the ability to stimulate the proliferation of CTLL-2 cells after binding to the SSTR2-expressing cells. SGC7901 cells, which express the same quantities of SSTR2 as SMMC7721 and H22 cells according to the Western blotting results, also have been used as test models, not only because of the expression of the SSTR receptors, but also because of their attaching growing behavior. During the CTLL-2 proliferating stimulation test, traces of IL- 2 or SIL should be removed by washing repeatedly since the $\mathrm{EC}_{50}$ of IL-2 is in the nanogram range. Compared with the cell group to which IL-2 was added, the groups of tumor cells that had SIL added could still stimulate the proliferation of CTLL cells, and this correlated with the dose after washing and changing the culture media. Those groups that had IL-2 added did not stimulate the proliferation of CTLL cells. The RGR of CTLL- 2 cells, which is a parameter used for distinguishing the experimental group on the same basis, showed a dramatic discrimination between the SIL and IL-2 groups. In order to appraise the affinity of the SIL binding to the SSTR through octreotide, a dose of octreotide that had been diluted sequentially had been simultaneously added to another group of tumor cells with SIL in order to 
test the competitive binding behaviors with the tumor target SIL. Although our experiments did not quantify the competitive behavior of octreotide or determine a dynamic binding mathematical model, the proliferation rate of the CTLL-2 cells that were stimulated by the residual binding of SIL on tumor cells exhibited a negative correlation with the appended dose of octreotide in the tumor cell culture. One possible conclusion might be that the bioactivity of SIL was not significantly altered after binding to the tumor cells through the appended octreotide on IL-2. SIL could exert the ability to stimulate the proliferation of the immunological cells at the tumor site if it could be delivered adequately to the tumor tissues, which means that more in vivo targeting studies and antitumor assays need to be performed in the future.

In summary, we have shown that the target immunocytokine SIL could be quantitatively produced with a high purity and natural bioactivity, and manufacturing on an industrial scale is feasible. The tumor targeting properties of SIL were first shown with in vitro cell models. Only those tumor cells that expressed SSTR, especially that of the 2 subtype, can be used for further in vivo tumor targeting and anti-tumor studies. Additional experiments, such as a targeting study and a half-life study performed in vivo and a combination with chemotherapy, are being designed in order to evaluate the efficacy of the recombinant targeting of chimeric immunocytokines.

\section{Acknowledgements}

This study was supported by Simcere-Medgenn Co., Ltd. We gratefully acknowledge the members of the technology centers for cooperation in the preparation of chimeric protein and for assistance with Mass spectrometry at the facilities of the Quality Control Department, as well as members of Zhou's laboratory in Tsinghua University for discussions and criticism throughout the course of this study.

\section{References}

1. Arap W, Pasqualini R and Ruoslahti E: Cancer treatment by targeted drug delivery to tumor vasculature in a mouse model. Science 279: 377-380, 1998.

2. Nanda PK, Lane SR, Retzloff LB, Pandey US and Smith CJ: Radiolabeled regulatory peptides for imaging and therapy. Curr Opin Endocrinol Diabetes Obes 17: 69-76, 2010.

3. Bussolati B, Grange C, Tei L, et al: Targeting of human renal tumor-derived endothelial cells with peptides obtained by phage display. J Mol Med 85: 897-906, 2007

4. Carter PJ: Potent antibody therapeutics by design. Nat Rev Immunol 6: 343-357, 2006.

5. Curnis F, Sacchi A, Borgna L, et al: Enhancement of tumor necrosis factor alpha Anti-tumor immunotherapeutic properties by targeted delivery aminopeptidase N(CD13). Nat Biotechnol 18: 1185-1190, 2000.

6. Schliemann C, Palumbo A, Zuberbuhler K, et al: Complete eradication of human B-cell lymphoma xenografts using rituximab in combination with the immunocytokine L19-IL2. Blood 113 2275-2283, 2009.

7. Halin C, Rondini S, Nilsson F, et al: Enhancement of the antitumor activity of interleukin-12 by targeted delivery to neovasculature. Nat Biotechnol 20: 264-269, 2002.

8. Ebbinghaus C, Ronca R, Kaspar M, et al: Engineered vasculartargeting antibody-interferon-gamma fusion protein for cancer therapy. Int J Cancer 116: 304-313, 2005.

9. Halin C, Gafner V, Villani ME, et al: Synergistic therapeutic effects of a tumor targeting antibody fragment, fused to interleukin 12 and to tumor necrosis factor alpha. Cancer Res 63: 3202-3210, 2003.
10. Melder RJ, Osborn BL, Riccobene T, et al: Pharmacokinetics and in vitro and in vivo anti-tumor response of an interleukin-2-human serum albumin fusion protein in mice. Cancer Immunol Immunother 54: 535-547, 2005.

11. Lotze MT, Matory YL, Ettinghausen SE, et al: In vivo administration of purified human interleukin 2. II. Half life, immunologic effects, and expansion of peripheral lymphoid cells in vivo with recombinant IL 2. J Immunol 135: 2865-2875, 1985.

12. Smith KA: IL-2. In: Cytokine Reference: A Compendium of Cytokines and Other Mediators of Host Defense. Oppenheim JJ and Feldman M (eds). Academic Press, New York, 2000.

13. Yang JC, Topalian SL, Schwartzentruber DJ, et al: The use of polyethylene glycol-modified interleukin-2 (PEG-IL-2) in the treatment of patients with metastatic renal cell carcinoma and melanoma. A phase I study and a randomized prospective study comparing IL-2 alone versus IL-2 combined with PEG-IL-2. Cancer 76: 687-694, 1995.

14. Marlind J, Kaspar M, Trachsel E, et al: Antibody-mediated delivery of interleukin-2 to the stroma of breast cancer strongly enhances the potency of chemotherapy. Clin Cancer Res 14: 6515-6524, 2008.

15. Weckbecker G, Lewis I, Albert R, Schmid HA, Hoyer D and Bruns C: Opportunities in somatostatin research: biological, chemical and therapeutic aspects. Nat Rev Drug Discov 2: 999-1017, 2003.

16. Kerros C, Cavey T, Sola B, Jauzac P and Allouche S: Somatostatin and opioid receptors do not regulate proliferation or apoptosis of the human multiple myeloma U266 cells. J Exp Clin Cancer Res 28: 77, 2009.

17. Schindler M, Holloway S, Humphrey PP, et al: Localization of the somatostatin sst2(a) receptor in human cerebral cortex, hippocampus and cerebellum. Neuroreport 9: 521-525, 1998.

18. Taniyama Y, Suzuki T, Mikami Y, Moriya T, Satomi S and Sasano H: Systemic distribution of somatostatin receptor subtypes in human: an immunohistochemical study. Endocr J 52: 605-611, 2005.

19. Strowski MZ and Blake AD: Function and expression of somatostatin receptors of the endocrine pancreas. Mol Cell Endocrinol 286: 169-179, 2008.

20. Reubi JC and Laissue JA: Multiple actions of somatostatin in neoplastic disease. Trends Pharmacol Sci 16: 110-115, 1995.

21. Reubi JC, Waser B, Schaer JC and Laissue JA: Somatostatin receptor sst1-sst5 expression in normal and neoplastic human tissues using receptor autoradiography with subtype-selective ligands. Eur J Nucl Med 28: 836-846, 2001.

22. Fjallskog ML, Ludvigsen E, Stridsberg M, Oberg K, Eriksson B and Janson ET: Expression of somatostatin receptor subtypes 1 to 5 in tumor tissue and intratumoral vessels in malignant endocrine pancreatic tumors. Med Oncol 20: 59-67, 2003.

23. de Herder WW, Hofland LJ, van der Lely AJ and Lamberts SW: Somatostatin receptors in gastroentero-pancreatic neuroendocrine tumours. Endocr Relat Cancer 10: 451-458, 2003.

24. Kumar U, Grigorakis SI, Watt HL, et al: Somatostatin receptors in primary human breast cancer: quantitative analysis of mRNA for subtypes 1-5 and correlation with receptor protein expression and tumor pathology. Breast Cancer Res Treat 92: 175-186, 2005.

25. Scarpignato $C$ and Pelosini I: Somatostatin analogs for cancer treatment and diagnosis: an overview. Chemotherapy 47 (Suppl 2): S1-S29, 2001.

26. Sun LC and Coy DH: Somatostatin receptor-targeted anti-cancer therapy. Curr Drug Deliv 8: 2-10, 2011.

27. Pyronnet S, Bousquet C, Najib S, Azar R, Laklai H and Susini C: Antitumor effects of somatostatin. Mol Cell Endocrinol 286: 230-237, 2008.

28. Sambrook J, Fritsch EF and Maniatis T (eds): Molecular Cloning: A Laboratory Manual. 2nd edition. Cold Spring Harbor Laboratory Press, New York, 1989.

29. Weston L: Measurement of interleukin-2 by bioassay. In: Interleukin Protocols. O'Neill LAJ and Bowie A (eds). Humana Press Inc., Totowa, pp186-192, 2001.

30. Yaciuk P: Co-immunoprecipitation of protein complexes. In: Adenovirus Methods and Protocols: Ad Proteins, RNA Lifecycle, Host Interactions, and Phylogenetics. Wold WS and Tollefson AE (eds). Vol 2, Human Press Inc., Totowa, pp103-111, 2007.

31. Anfinsen CB and Haber E: Studies on the reduction and re-formation of protein disulfide bonds. J Biol Chem 236: 1361-1363, 1961. 